\title{
KULEUVEN
}

\section{Traditional starter cultures for enset fermentation: Unravelling their production and microbial composition}

\author{
A.F. Andeta ${ }^{1,2,3}$, E. Gorrens ${ }^{1,2}$, D. Vandeweyer ${ }^{1,2}$, K. Vancampenhout ${ }^{1}$, L. Van Campenhout ${ }^{1,2}$
}

${ }^{1} \mathrm{KU}$ Leuven, Campus Geel, Lab4Food, Technology Cluster Bioengineering Technology, Department of Microbial and Molecular Systems, Geel, Belgium ${ }^{2}$ KU Leuven, Leuven Food Science and Nutrition Research Centre (LFoRCe), Leuven, Belgium

${ }^{3}$ Arba Minch University, College of Natural Sciences, Department of Biology, Arba Minch, Ethiopia

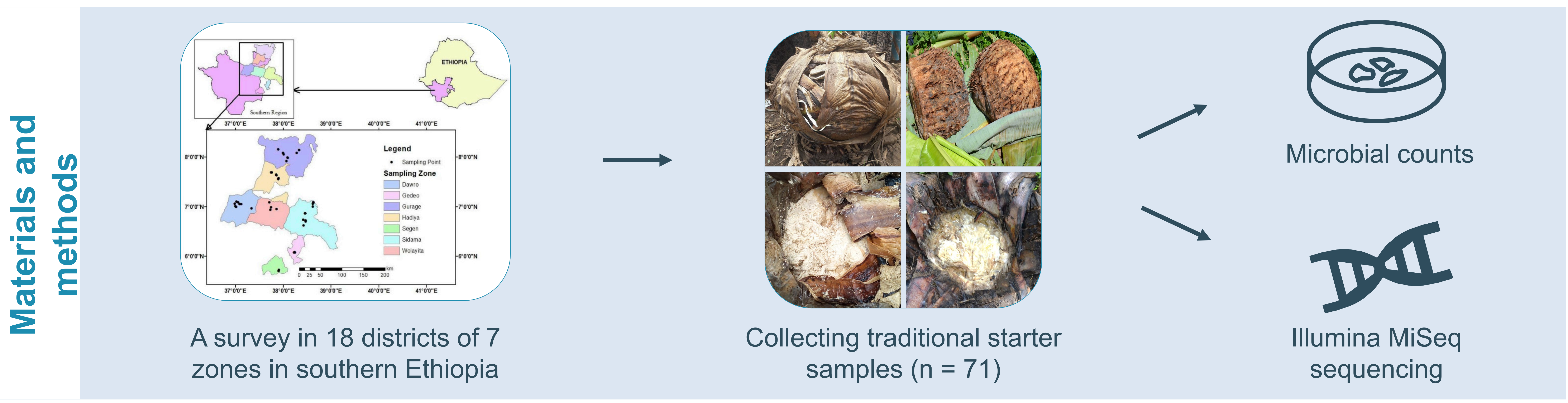

$\mathrm{pH}$
- $\mathrm{pH}$ value ranging from $3.68 \pm 0.16$ to $5.62 \pm$ 0.33 (individual districts).

- Significant differences due to different preparation procedures.
Table $1 \mathrm{pH}$ of traditional starters made for enset superscripts indicate significant differences $(p<0.05)$. Sidama Wolayita Dawro Segen Gedeo Hadiya Gurage
$4.84 \pm 0.09^{b}$

$4.60 \pm 0.09^{b, c, d}$

$3.80 \pm 0.10^{\mathrm{e}}$

$4.94 \pm 0.39 a, b, c, d$

$5.62 \pm 0.33^{a}$

$4.30 \pm 0.25^{d, e}$ $4.20 \pm 0.38^{c, d, e}$ fermentation. For the zones, means and standard

\section{Microbial counts}

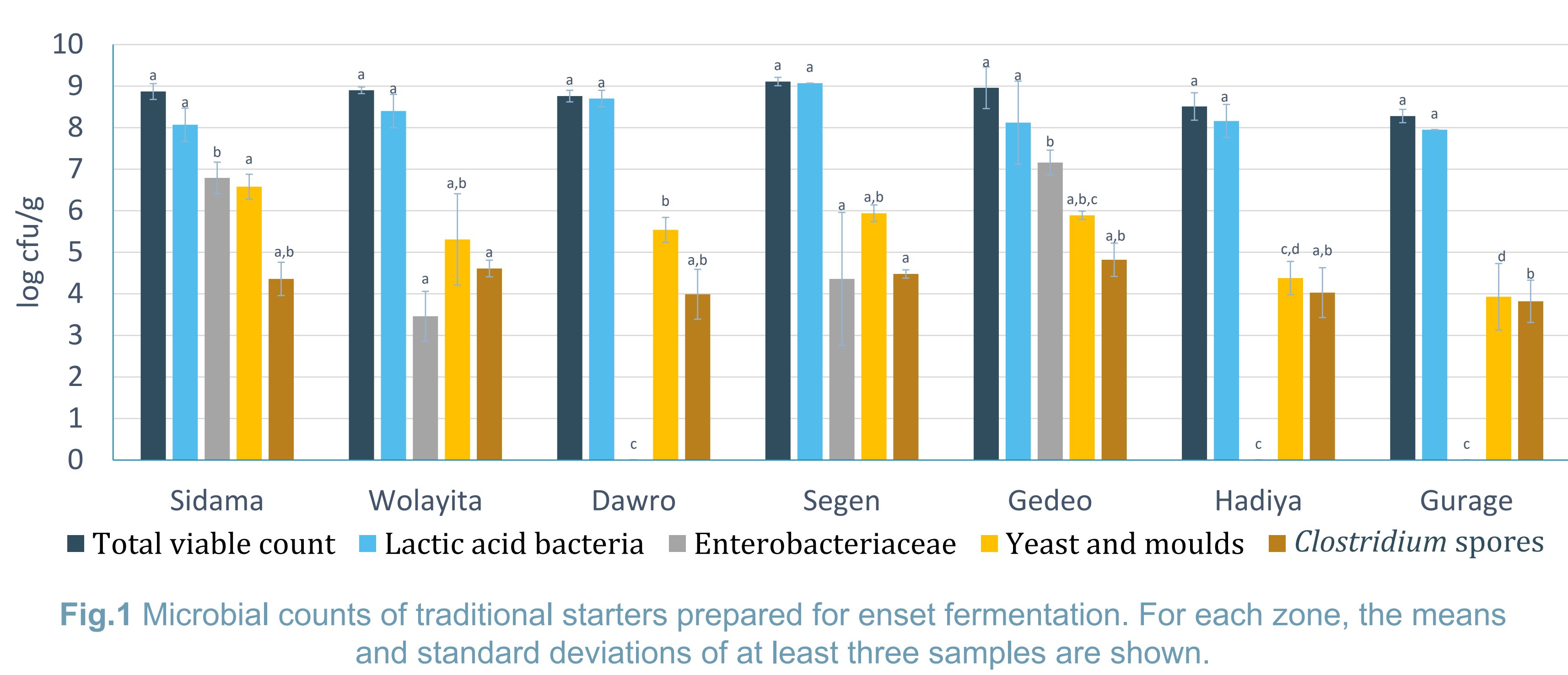

- High numbers of lactic acid bacteria (LAB).

- Enterobacteriaceae were identified in some samples.

- Clostridium spores were present in all traditional starters. The presence of this genus can include safety risks.

\section{Bacterial community composition}

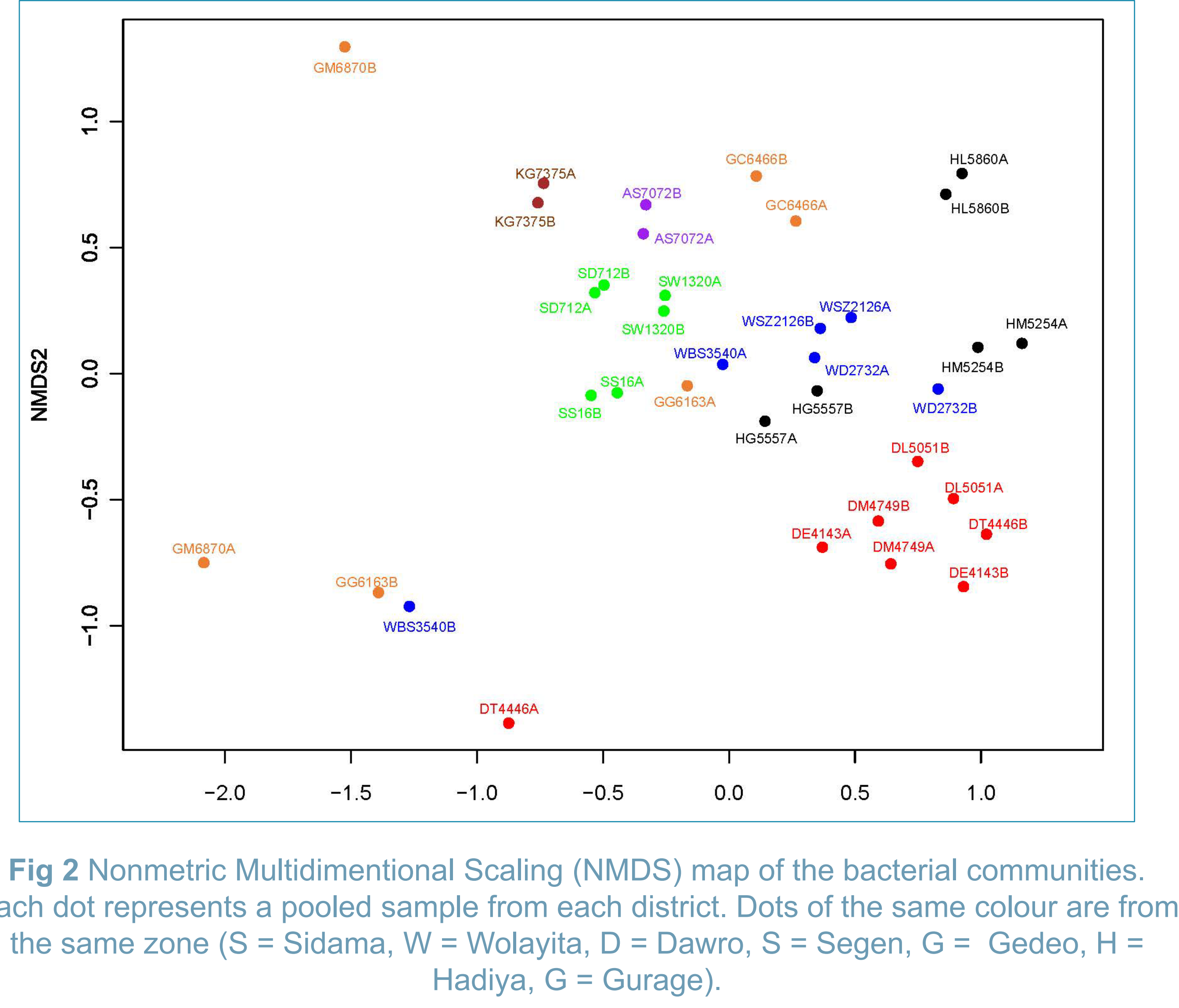

- Most samples from the same zone have a similar bacterial community.

- Most abundant phylum: Firmicutes > Bacteroidetes > Proteobacteria.

- Lactobacillus, Lactococcus and Leuconostoc were the dominant $L A B$.

- These isolates will be further considered for the development of a commercial enset starter culture. 